\title{
Effective hydraulic conductivity and diffusivity of randomly heterogeneous porous solids with compressible constituents
}

\author{
Tobias M. Müller ${ }^{a}$ \\ Geophysikalisches Institut, Universität Karlsruhe, Hertzstr. 16, 76187 Karlsruhe, Germany \\ Boris Gurevich ${ }^{\text {b) }}$ \\ Department of Exploration Geophysics, Curtin University and CSIRO Petroleum, G.P.O. Box U1987 Perth, \\ WA 6845, Australia
}

(Received 14 November 2005; accepted 27 February 2006; published online 24 March 2006)

\begin{abstract}
Inhomogeneous fluid-saturated porous materials are often probed with diffusion waves to estimate their effective hydraulic conductivity and diffusivity. Analysis of diffusion wave fields in randomly inhomogeneous poroelastic structures provides new insight into how fluctuations of the compressible constituents affect the effective diffusivity. Based on the method of statistical smoothing an effective wave number of the coherent diffusion wave field is computed. From this wave number both an effective hydraulic conductivity and diffusivity are identified. These transport properties are frequency dependent. The correspondence between this conductivity and that estimated from unsteady flow through porous media based on Darcy's law is elucidated. () 2006 American Institute of Physics. [DOI: 10.1063/1.2189455]
\end{abstract}

Probing complex structures with diffusion waves has become a powerful technique in various physical disciplines. ${ }^{1}$ In particular, diffusion waves are used to characterize transport properties of fluid-saturated porous solids. ${ }^{2}$ If the porous material has a deformable frame, the transport properties can be affected by the compressibility of the solid as well as the fluid phase. This effect can be analyzed using Biot's theory of poroelasticity. ${ }^{3}$ In particular, Biot's theory predicts the existence of so-called Biot's slow wave, which in the lowfrequency (i.e., quasistatic) limit is governed by the diffusion equation. ${ }^{4}$ The corresponding diffusion coefficient depends on compressibilities of the fluid and solid phases as well as permeability and fluid viscosity.

Transport properties of porous materials are particularly affected by spatial heterogeneity. The study of these effects usually employs the concept of random media, and requires an analysis of field equations with random coefficients. One method that can be applied in this context is method of statistical smoothing, ${ }^{5}$ which has been widely used in the analysis of wave propagation in random media as well as flow through random rigid porous media. ${ }^{6,7}$

In this letter we will employ the method of statistical smoothing to compute the effective hydraulic diffusivity of randomly inhomogeneous porous media. The medium is assumed to be governed by the low-frequency version of Biot's equations of poroelasticity where the slow compressional wave is a diffusion wave characterized by the wave number

$$
k_{0}=\sqrt{i \omega / D_{0}}
$$

with hydraulic diffusivity $D_{0} \cdot{ }^{8}$ Neglecting the interaction with other wave modes, we analyze the coherent diffusion wave field only. We derive an expression for the effective diffusion wave number from which we extract the effective, frequency-dependent transport properties. There exists low and high frequency regimes depending an whether the diffu-

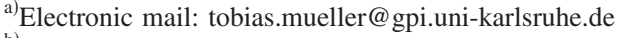

${ }^{b)}$ Electronic mail: boris.gurevich@geophy.curtin.edu.au
}

sion wavelength $2 \pi / k_{0}$ is large or small compared to the spatial scale of the inhomogeneities.

In random poroelastic media all parameters are represented by random fields of the form $X=\bar{X}+\widetilde{X}=\bar{X}\left(1+\varepsilon_{X}\right)$, where $\bar{X}$ is a constant background value and $\tilde{X}(\mathbf{r})$ is the fluctuating part. Parameter $\varepsilon_{X}=\tilde{X} / \bar{X}$ denotes the relative fluctuations and has zero mean $\left(\left\langle\varepsilon_{X}\right\rangle=0\right)$, autocorrelation function $B_{X X}(\delta \mathbf{r})=\left\langle\varepsilon_{X}(\mathbf{r}+\delta \mathbf{r}) \varepsilon_{X}(\mathbf{r})\right\rangle$, and variance $\left\langle\varepsilon_{X}^{2}\right\rangle=B_{X X}(0)=\sigma_{X X}^{2}$.

The starting point of our analysis is the poroelastic Dyson integral equation for the mean Green's function of Biot's equations ${ }^{9}$ in the random porous medium. By neglecting all contributions from the fast compressional and shear waves, we can write Dyson's equation for the matrix containing the mean Green's tensors $\overline{\mathbf{G}}$ in the form

$$
\overline{\mathbf{G}}=\mathbf{G}_{0}+\iint \mathbf{G}_{0} \mathbf{Q} \overline{\mathbf{G}},
$$

where $\mathbf{G}_{0}$ denotes the matrix of Green's function for the homogeneous background ${ }^{9}$

$$
\mathbf{G}_{0}=\frac{\kappa_{0}}{4 \pi i \omega} \partial_{i} \partial_{j} \frac{\exp \left(i k_{0} R\right)}{R}\left[\begin{array}{cc}
-\frac{C^{2}}{H^{2}} & \frac{C}{H} \\
\frac{C}{H} & -1
\end{array}\right],
$$

where $R$ denotes the distance from source to observation point and $\partial_{i}$ denotes partial spatial derivative. $\mathbf{Q}$ is the matrix of the kernel-of-mass operators

$$
\mathbf{Q}=\left\langle\widetilde{\mathbf{L}} \mathbf{G}_{0} \widetilde{\mathbf{L}}+\int \widetilde{\mathbf{L}} \mathbf{G}_{0} \widetilde{\mathbf{L}} \mathbf{G}_{0} \tilde{\mathbf{L}}+\int \ldots\right\rangle,
$$

where $\widetilde{\mathbf{L}}$ denotes the matrix of the perturbing operators 


$$
\tilde{\mathbf{L}}=\left[\begin{array}{cc}
\partial_{i} \tilde{H} \partial_{j} & \partial_{i} \tilde{C} \partial_{j} \\
\partial_{i} \tilde{C} \partial_{j} & i \omega \tilde{p} \delta_{i j}+\partial_{i} \tilde{M} \partial_{j}
\end{array}\right]
$$

with the identity tensor $\delta_{i j}$. The method of statistical smoothing consists now in truncating $\mathbf{Q}$ after the first term. In Eqs. (3) and (5) $H$ is the undrained, low-frequency $P$-wave modulus given by Gassmann's equation $H=P_{d}+\alpha^{2} M$, where $M$ is the pore space modulus $M=\left[(\alpha-\phi) / K_{g}+\phi / K_{f}\right]^{-1}$ and $P_{d}$ $=K_{d}+4 / 3 \mu$ is the $P$-wave modulus of the drained frame, $\alpha$ $=1-K_{d} / K_{g}$ is the Biot-Willis coefficient, $C=\alpha M . K_{g}, K_{d}$, and $K_{f}$ denote the bulk moduli of the solid phase, the drained frame, and the fluid phase, while $\mu$ denotes the porousmaterial shear modulus. In Eq. (3) the conductivity is denoted as $\kappa_{0}$ while in Eq. (5) $\widetilde{p}$ denotes the fluctuating part of the reciprocal conductivity $p=1 / \kappa_{0}$. The diffusivity in Eq. (1) can be expressed through $D_{0}=\kappa_{0} N$, where $N=M P_{d} / H$.

Equation (2) contains a double convolution which in the spatial Fourier domain yields a set of algebraic equations. Retaining only terms of order $O\left(\epsilon^{2}\right)$ a simpler equation for the [2,2] component of $\overline{\mathbf{G}}$ is obtained,

$$
\bar{g}=g_{0}+\left(8 \pi^{3}\right)^{2} g_{0} q \bar{g},
$$

where $g_{0}$ and and $q$ are the Fourier transforms of the corresponding components of $\mathbf{G}_{0}$ and $\mathbf{Q}$, respectively. Assuming that the mean Green's function $\bar{g}$ is of the same functional form as $g_{0}$, but involving an effective wave number $k^{*}$, we can solve Eq. (6) for $k^{*}$. The truncated kernel-of-mass operator matrix element $q$ can be evaluated for statistically isotropic random media and yields the following approximation for the square of the effective wave number:

$$
k^{* 2}=k_{0}^{2}\left[1+\Delta_{s} \xi(\omega)\right],
$$

where

$$
\xi(\omega)=1+k_{0}^{2} \int_{0}^{\infty} r B(r) \exp \left(i k_{0} r\right) d r
$$

and

$$
\Delta_{s}=\left\langle\left(\frac{\alpha^{2} M}{P_{d}} \varepsilon_{\alpha}-\varepsilon_{K_{f}}+\varepsilon_{\phi}\right)^{2}\right\rangle+\frac{\sigma_{p p}^{2}}{3} .
$$

We note that $B(r)$ in Eq. (8) is defined as the normalized autocorrelation function such that $B_{X Y}=\sigma_{X Y}^{2} B(r)$ with $B(0)$ $=1$. The method of statistical smoothing is precise for weak fluctuations only, and therefore, Eq. (7) is applicable if $\Delta_{s}$ $\lesssim 1$. The physical interpretation of the effective diffusion wave number is straightforward. Due to multiple scattering, or in terms of diffusion wave terminology, ${ }^{1}$ due to accumulation and depletion processes at randomly spaced inhomogeneities, an initially homogeneous diffusion wave with $k_{0}$ becomes at finite frequencies an inhomogeneous diffusion wave characterized by $k^{*}$ (i.e., $\mathfrak{R}\left\{k^{*}\right\} \neq \Im\left\{k^{*}\right\}$ ). In the limits of zero and infinite frequency the diffusion wave becomes homogeneous again with the effective wave numbers $k^{*}(\omega$ $\rightarrow 0)=k_{0}\left(1+\Delta_{s}\right)$ and $k^{*}(\omega \rightarrow \infty)=k_{0}$, respectively. The frequency dependence of this phenomenon is illustrated in Fig. 1 .

Effective wave number (7) can be used to construct approximation of the effective transport properties. Assuming that the effective wave number $k^{*}$ involves an effective hy-

draulic diffusivity $D^{*}$ such that $k^{*}=\sqrt{i \omega / D^{*}}$, we obtain model. Shown is the real part of $D^{*}$ for varying $\Delta_{s}$.
Downloaded 04 Apr 2006 to 129.13.72.153. Redistribution subject to AlP license or copyright, see http://apl.aip.org

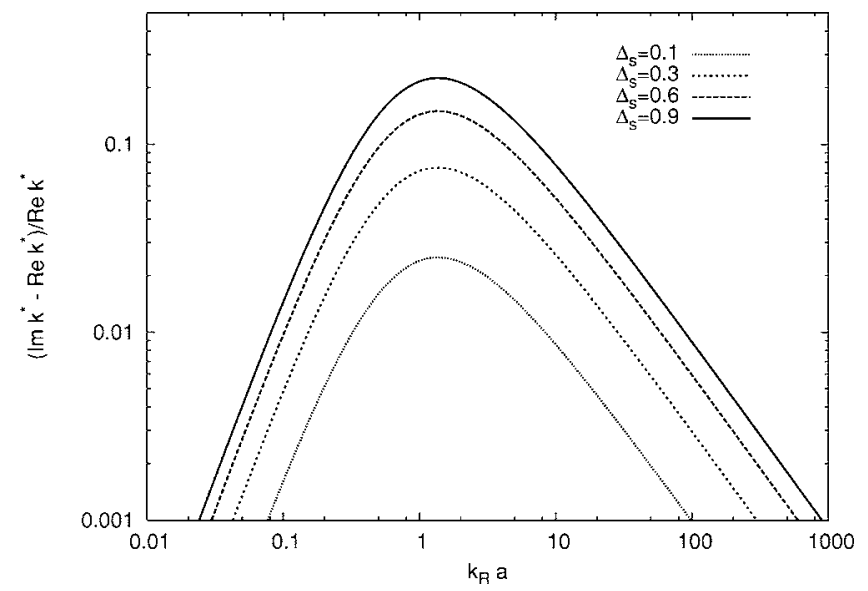

FIG. 1. Normalized difference of real and imaginary part of the effective wave number vs frequency for varying $\Delta_{s}$.

$$
D^{*}=D_{0}\left[1-\Delta_{s} \xi(\omega)\right] \text {. }
$$

Figure 2 illustrates the frequency dependence of this effective diffusivity for varying $\Delta_{s}$ in a model of a porous sandstone where the inhomogeneities are statistically characterized by a correlation function of the form $B(r)=\exp ($ $-|r| / a$ ), where $a$ is the correlation length (shown is the real part of $D^{*}$ as a function of the dimensionless frequency $k_{R} a$, where $k_{R}$ denotes the real part of $k_{0}$ ).

As can be seen from Fig. 2, the presence of inhomogeneities reduces the effective diffusivity below the background diffusivity. In the zero frequency limit we have $D^{*}(\omega \rightarrow 0)=D_{0}\left(1-\Delta_{s}\right)$, whereas for infinitely high frequency the background value is obtained, $D^{*}(\omega \rightarrow \infty)=D_{0}$. The magnitude of the diffusivity dispersion is controlled by $\Delta_{s}$ which contains the second order moments of the random fields of $\alpha$, $\phi, K_{f}$, and $p$. The role of cross correlations is particularly interesting. For example, negative cross correlation between the Biot-Willis coefficient and the fluid bulk modulus, i.e., if there is a stiff fluid in the pore-space of a very compressible porous solid, produces an enhanced diffusivity dispersion.

Analogously to $D^{*}$, we can construct an effective hydraulic conductivity $\kappa^{*}$ by assuming that $k^{*}$ is of the form $k^{*}=\sqrt{i \omega / \kappa^{*} N}$, i.e., $N$ is constant. In this case the calculations outlined above can be performed in all space dimensions and we obtain

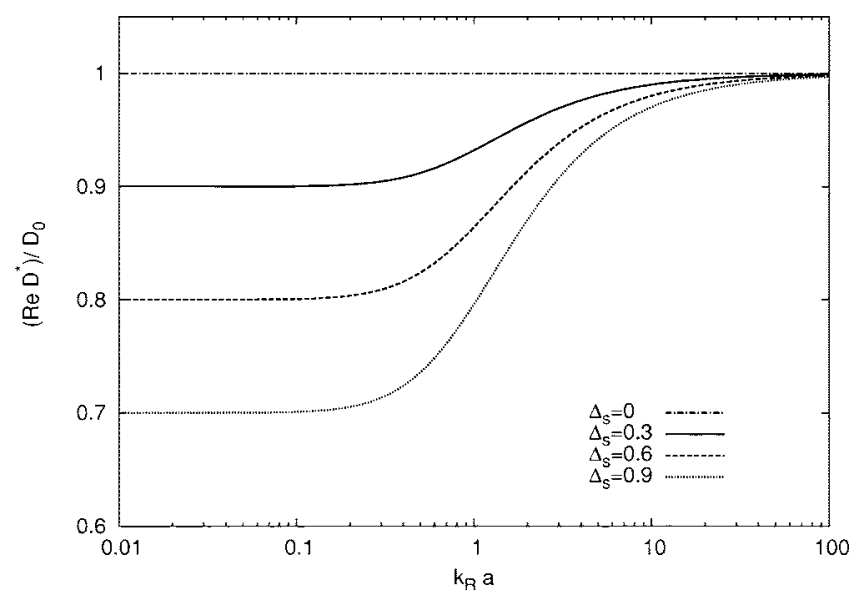

FIG. 2. Normalized effective diffusivity vs frequency for a porous sandstone model. Shown is the real part of $D^{*}$ for varying $\Delta_{s}$. 


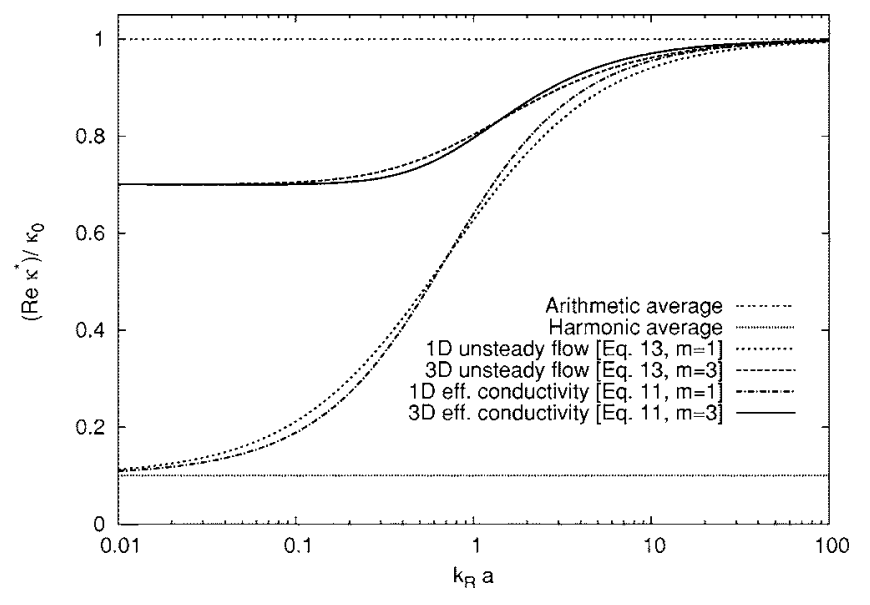

FIG. 3. Normalized effective conductivity in 1D and 3D space vs frequency. Shown are the real parts of $\kappa^{*}$ and $\kappa_{\text {flow }}^{*}$ according to Eqs. (11) and (13) with $\sigma_{p p}^{2}=0.9$.

$$
\kappa^{*}=\kappa_{0}\left[1-\frac{\sigma_{p p}^{2}}{m}\left(1-4 \int \frac{k_{R}^{4}}{4 k_{R}^{4}+K^{4}} \Phi(K) d \mathbf{K}\right)\right]
$$

in $m$-dimensional space $(m=1,2$ or 3$)$ where $\Phi(K)$ denotes the fluctuation spectrum. This result is displayed in Fig. 3. The effective hydraulic conductivity is bounded by

$$
\kappa_{H} \leqslant \kappa^{*} \leqslant \kappa_{A},
$$

where $\kappa_{H}$ and $\kappa_{A}$ denote the harmonic and arithmetic averages, respectively. In the low-frequency limit the lower bound becomes an identity for $m=1$ only, whereas at infinitely high frequencies the upper bound is reached exactly in all space dimensions.

For a poroelastic continuum the diffusion wave mode (Biot's slow wave) in the limit of zero frequency is equivalent to the quasistatic flow. ${ }^{4}$ It is therefore interesting to compare our results with estimates of an effective hydraulic conductivity in random porous media based on Darcy's law. ${ }^{7,10}$ Time dependent, i.e., unsteady, flow analyzed on the basis of Darcy's law in conjunction with the time-dependent continuity equation yields a diffusion equation for the pore pressure of the form $S \partial_{t} P=\nabla \cdot(\kappa \nabla P)$, where $S$ is the specific storativity. $^{11,12}$ In statistically isotropic $m$-dimensional random media an averaged Darcy law allows us to define an effective hydraulic conductivity. The latter can be explicitly computed for weakly inhomogeneous structures involving only the spatial correlation of the conductivity fluctuations, ${ }^{12}$

$$
\kappa_{\text {flow }}^{*}=\kappa_{0}\left[1-\frac{\sigma_{\kappa \kappa}^{2}}{m} \int \frac{K^{2}}{k_{R}^{2}+K^{2}} \Phi(K) d \mathbf{K}\right] .
$$

This effective conductivity is also displayed in Fig. 3. Note that the low and high frequency limits as well as the inflection points of $\kappa^{*}$ and $\kappa_{\text {flow }}^{*}[$ Eqs. (11) and (13)] are identical. The slightly different effective conductivities according to Eqs. (11) and (13) are due to the use of two different governing equations. The present analysis is based on Biot's equations of dynamic poroelasticity, whereas Eq. (13) results from a diffusion equation for the pore pressure which in turn is obtained from a quasistatic approximation. Also, both approaches show that in the weak fluctuation case the effective conductivity does not depend on the compressibilities of the porous material and the fluid phase.

In conclusion, the main result of this letter is the expression for the effective hydraulic diffusivity (10). It depends not only on the the second-order statistics of the conductivity but also on that of the poroelastic moduli. Therefore, for accurate estimation of the effective hydraulic conductivity from diffusion wave characteristics, fluctuations of the compressibilities of the porous material must be accounted for.

This work was kindly supported by the Deutsche Forschungsgemeinschaft (Contract No. MU 1725/1-3).

${ }^{1}$ A. Mandelis, Diffusion-Wave Fields (Springer, New York, 2001).

${ }^{2}$ J. R. Rice and M. P. Cleary, Rev. Geophys. Space Phys. 14, 227 (1976).

${ }^{3}$ M. A. Biot, J. Appl. Phys. 33, 1482 (1962).

${ }^{4}$ R. N. Chandler and D. L. Johnson, J. Appl. Phys. 52, 3391 (1981).

${ }^{5}$ F. C. Karal and J. B. Keller, J. Math. Phys. 5, 537 (1964).

${ }^{6}$ P. R. King, J. Phys. A 20, 3935 (1987).

${ }^{7}$ J. B. Keller, Transp. Porous Media 43, 395 (2001).

${ }^{8}$ A. N. Norris, J. Acoust. Soc. Am. 77, 6 (1985); 77, 2012 (1985).

${ }^{9}$ T. M. Müller and B. Gurevich, J. Acoust. Soc. Am. 117, 4 (2005); 117, 1796 (2005).

${ }^{10}$ D. T. Hristopulos and G. Christakos, Phys. Rev. E 55, 7288 (1997).

${ }^{11}$ G. Dagan, Water Resour. Res. 18, 1571 (1982).

${ }^{12}$ P. Indelman, J. Fluid Mech. 310, 39 (1996). 